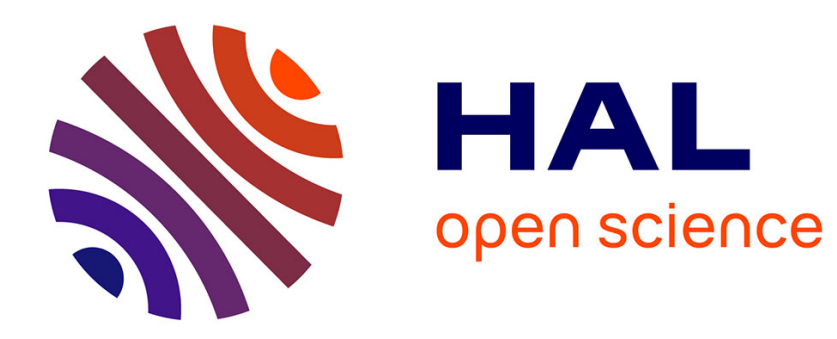

\title{
La possibilité d'une ville. Comprendre les spatialités homosexuelles en milieu urbain
}

Stéphane Leroy

\section{To cite this version:}

Stéphane Leroy. La possibilité d'une ville. Comprendre les spatialités homosexuelles en milieu urbain. Espaces et sociétés (Paris, France), 2009, 139, pp.159-174. hal-00447807

\section{HAL Id: hal-00447807 \\ https://hal.science/hal-00447807}

Submitted on 15 Jan 2010

HAL is a multi-disciplinary open access archive for the deposit and dissemination of scientific research documents, whether they are published or not. The documents may come from teaching and research institutions in France or abroad, or from public or private research centers.
L'archive ouverte pluridisciplinaire HAL, est destinée au dépôt et à la diffusion de documents scientifiques de niveau recherche, publiés ou non, émanant des établissements d'enseignement et de recherche français ou étrangers, des laboratoires publics ou privés. 


\title{
La possibilité d'une ville. Comprendre les spatialités homosexuelles en milieu urbain
}

\author{
Stéphane Leroy \\ EA3482 LAB'URBA \\ Université Paris-Est Créteil
}

\begin{abstract}
Résumé
La grande ville occupe en Occident une place particulière dans l'imaginaire et la culture gays. Elle est attractive pour les homosexuels car elle leur garantit à la fois l'anonymat et la visibilité et augmente les possibilités de rencontre. Elle permet surtout l'identification à des territoires spécifiques voire la construction d'une identité collective, notamment par le développement de réseaux de sociabilité qui s'appuient sur un tissu dense de commerces et d'associations. Enfin, elle offre une plus grande liberté que les autres espaces par rapport aux contraintes quotidiennes de l'hétéronormativité. Cependant, comme ailleurs, cette liberté a des limites et les homosexuels doivent constamment ajuster leur comportement en fonction des lieux et trouver la distance adéquate afin d'échapper à la stigmatisation.
\end{abstract}

Mots-clés : distance, homosexualités, norme, ville, visibilité.

\begin{abstract}
Large cities have a special position in gay imagination and culture in Western countries. Homosexuals feel them attractive because they guarantee both anonymity and visibility, and increase the possibilities of meeting their fellows. Large cities allow homosexuals to especially identify with specific territories, notably to develop a collective identity, mainly by the expansion of social networks based on a lot of gay shops and associations. Evidently therefore, these cities give more freedom to homosexuals than other spaces in terms of the daily constraints of the heteronormativity. However, as anywhere, this freedom has its limits. Homosexuals must constantly adapt their behaviour depending on the places, and find the appropriate measure of discretion in order to avoid stigmatization.
\end{abstract}

Keywords : distance, homosexualities, norm, city, visibility.

\section{Resumen}

En Occidente, la gran ciudad ocupa un lugar particular en el imaginario y la cultura gay. Es atractiva para los homosexuales porque les garantiza a la vez anonimato y visibilidad, y aumenta las posibilidades de encuentro. Permite sobre todo la definición a territorios específicos 
o incluso la construcción de una identidad colectiva, en particular por el desarrollo de redes de sociabilidad que se basan en un tejido denso de comercios y asociaciones. Ella ofrece una mayor libertad que los otros espacios con relación a las dificultades diarias de la heteronormatividad. Sin embargo, esta libertad tiene límites y los homosexuales deben constantemente ajustar su comportamiento en función de los lugares y encontrar la distancia adecuada afín de escaparse a la estigmatización ;

Palabras claves : distancia, homosexualidades, norma, ciudad, visibilidad. 
Depuis quelques décennies, des recherches sur la population homosexuelle se sont développées en géographie, d'abord aux États-unis, où le travail de Manuel Castells (1983) sur les spatialités gays à San Francisco apparaît comme pionnier, ensuite dans les autres pays anglo-saxons, et enfin partout ailleurs dans les pays développés. Mais aujourd'hui encore, la France, comme d'autres pays, accuse un grand retard sur ces questionnements. Ces recherches, comme celles en histoire notamment (Aldrich, 2006), montrent que les lesbiennes et surtout les gays entretiennent un rapport privilégié avec la ville dans les pays occidentaux (Binnie et Valentine, 1999). Si George Chauncey (2003) montre avec l'exemple de New York qu'une «ville gay» exista pendant longtemps à l'intérieur d'une ville hétérosexuelle mais à son insu, les homosexuels ont progressivement acquis un droit à la visibilité voire à la centralité dans certains espaces urbains. Il se manifeste par l'appropriation de quartiers et par le dévoilement de soi lors de manifestations revendicatives et festives dans l'espace public. Ce lien privilégié concerne presque exclusivement la grande ville, sans qu'il soit possible d'indiquer un seuil de population, tant les spécificités nationales jouent ${ }^{1}$, et seulement dans les pays où l'homosexualité n'est pas réprimée.

Cet article interroge l'apport de la recherche géographique, en particulier anglosaxonne, pour comprendre les formes de la relation qui existe entre les homosexuels et la ville et expliquer pourquoi la ville est l'espace dans lequel ils sont aujourd'hui les plus visibles. L'articulation entre visibilité et invisibilité est fondamentale dans les pratiques et les représentations de l'espace des homosexuels. Si les spatialités lesbiennes ne sont pas oubliées (mais elles mériteraient un article spécifique), on s'attache plus particulièrement aux gays, sûrement plus nombreux dans les villes (Gates et Ost, 2004) et nettement plus visibles. Ce texte souligne aussi l'intérêt de questionner et d'analyser les spatialités des homosexuels pour enrichir les connaissances géographiques sur la ville. Comparée au foisonnement des travaux anglo-saxons, la géographie française apparaît bien silencieuse. Il faut essayer d'en comprendre les raisons. Puis, on présente et explique les différentes approches de la géographie urbaine des homosexualités, ce qui n'a jamais été fait dans la littérature géographique de langue française. Elles montrent l'importance de la grande ville pour les homosexuels, notamment comme espace de sécurité et d'affirmation identitaire relatives, et donnent à voir différents types de spatialités : des quartiers commerciaux appropriés par les homosexuels masculins à leurs lieux de drague, en passant par les territorialisations éphémères de l'espace public. Elles questionnent les modalités de la visibilité, mais

1. Dans la suite du texte, sauf précision contraire, il faut donc lire «grande ville » lorsque est écrit « ville ». 
aussi la norme et sa transgression. Enfin, la question de savoir s'il existe encore des territoires interdits aux gays et aux lesbiennes dans la ville est posée. Ne développent-ils pas quotidiennement différentes stratégies pour la pratiquer et contourner les formes normatives qui la structurent?

\section{LES (HOMO)SEXUALITÉS OUBLIÉES DE LA GÉOGRAPHIE FRANÇAISE}

Il est important de s'arrêter sur la place de cette thématique dans la géographie française. Objet difficile à appréhender et à définir, l'homosexualité y demeure largement ignorée, dans un contexte académique qui continue de privilégier une approche universaliste de la société.

\section{Un objet exotique et flou}

S'il est acquis que nombre de constructions identitaires, individuelles et collectives, s'appuient sur l'espace pour s'élaborer (Di Méo, 2004), la dimension sexuelle de ces constructions est rarement envisagée dans la géographie française. Ce silence est partagé par d'autres sciences sociales, comme l'histoire notamment (Tamagne, 2006). Les travaux sur le genre d'auteurs majeurs comme Gayle Rubin ou Judith Butler ont mis de nombreuses années à être traduits en français. C'est d'autant plus surprenant que les spécialistes américains du genre convoquent à l'envie de nombreux penseurs français, tels que Baudrillard, Deleuze, Derrida, Foucault, Wittig, etc. (Cusset, 2003). Si la géographie française s'est récemment ouverte à cette thématique, son développement demeure fragile, encore largement méconnu voire ignoré ou méprisé. Travailler sur les spatialités des sexualités, en particulier sur celles des homosexualités, apparaît encore en France comme original voire exotique et ceux qui s'y consacrent doivent fréquemment se justifier.

Certes, il est compliqué de travailler sur un objet difficile à saisir. La définition de ce que l'on entend par population homosexuelle n'est pas simple, car l'orientation sexuelle est loin d'être une réalité stable dans le temps (Butler, 2005). Même s'il s'agit d'une séparation un peu schématique et qu'il existe des situations intermédiaires, une suggestion est de différencier, d'une part les individus qui se définissent voire se revendiquent comme homosexuels, qui partagent - à un moment donné de leur existence - une identité collective et une culture commune et dont les territorialités sont visibles ; et d'autre part, les individus qui ont eu ou ont épisodiquement des rapports sexuels avec des individus de même sexe, mais qui ne fondent pas une partie de leur identité là dessus et/ou qui se définissent comme hétérosexuels. Ils n’ont pas de pratiques spatiales spécifiques à l'homosexualité, sauf pour ceux qui fréquentent les 
espaces publics détournés de leur usage à des fins d'interactions sexuelles. On se focalise ici sur les premiers, appelés gays quand il s'agit d'hommes et lesbiennes quand il s'agit de femmes. Dans le monde anglo-saxon, le terme de gay a progressivement remplacé celui d'homosexuel, jugé trop médical et renvoyant seulement à une préférence sexuelle.

\section{Un objet sans importance?}

On peut penser que le milieu académique français des sciences sociales privilégie une conception universaliste de la société, ce qui lui fait rejeter la pertinence d'une analyse spécifique des minorités. Sans nier l'existence d'inégalités et de différences, cette conception amène à rechercher des explications qui les transcendent. Dans ce contexte, «l'universalisme peut devenir la stratégie de lutte d'un groupe dominant [les hétérosexuels] qui prétend en libérer un autre [les homosexuels] des chaînes particularistes auxquelles il semble s'accrocher» (Frioux, 2003, p. 413). L'universalisme devient synonyme d'hétérosexisme ${ }^{2}$ et sert de tactique pour empêcher toute expression de la différence et toute évolution du droit, comme les débats sur le Pacs l'ont montré. Cette stratégie témoigne de l'embarras de certains face à la question de l'homosexualité car elle remet en cause le pouvoir du groupe dominant et les normes qu'il impose. L'universalisme et l'hétérosexisme produisent des fantasmes, comme celui de l'existence d'un ghetto gay, ghetto dont le sens et l'usage ont glissé de l'entité spatiale à la pratique sociale (Leroy, 2005), et annihilent toutes réflexions sur les normes que les représentations et pratiques spatiales des gays et des lesbiennes révèlent et contestent (Cattan et Leroy, 2008).

Enfin, beaucoup de géographes français, privilégiant des analyses de rapports de force d'une autre nature, considèrent que la sexualité n'est pas un objet scientifique digne d'intérêt (Blidon, 2008). Ainsi, en 1998, Philippe Pinchemel déclare dans Historiens et Géographes:

«Je voyais l'autre jour dans une revue américaine de géographie que l'on vient de créer un groupe sur la sexualité et l'espace géographique. On en est maintenant à traiter géographiquement de l'homosexualité et de l'hétérosexualité ! Nous n'en sommes pas là en France ; même la "géographie féminine" (féministe ?) n'a pas traversé la Manche, alors que la gender geography est très florissante outreManche ; mais cela ne saurait tarder, avec le Tunnel... $»^{3}$.

2. «L'hétérosexisme désigne un système de domination qui hiérarchise les sexualités et fait de l'hétérosexualité la marque exclusive de la normalité. (...) Par ailleurs, la puissance du système hétérosexiste est relayée par le pouvoir normatif de l'hétérosexualité ("hétéronormativité") et l'imprégnation du modèle hétérosexuel dans la culture occidentale, notamment dans les sciences humaines ("hétérocentrisme") » (Rocchi, 2003, p. 243)

3. PINCHEMEL, P. 1998. «Etudier la face de la terre », entretien, Historiens et Géographes, n³67. 
Et en 2003, lors d'un débat sur le postmodernisme dans L'Espace géographique, Roger Brunet s'interroge :

«Finalement, il est peut-être assez légitime, et facile à expliquer, que les géographes ne se soient pas beaucoup intéressés jusqu'ici, par exemple à l'homosexualité, aux raves parties ou à la musique sérielle. Est-ce que nous avons des choses pertinentes à dire sur de tels sujets ? ${ }^{4}$.

Pourtant, l'un des apports les plus intéressants des analyses qui questionnent les sexualités est de montrer que l'espace n'est ni asexué ni asexuel et que la sexualité n'est pas non plus a-spatiale (Bell et Valentine, 1995a). L'espace public en particulier, est une construction sociale dominée par les hommes (Bondi et Rose, 2003) et il caractérisé depuis toujours par l'hétérosexisme. Toutefois, lorsque la géographie questionne les sexualités, c'est presque toujours des homosexualités, qu'elles soient féminines ou masculines, dont il s'agit. La géographie des hétérosexualités n'existe quasiment pas (Hubbard, 2000). Est-ce parce que pendant longtemps l'ensemble de la géographie humaine s'apparentait à une géographie de l'hétérosexualité ? De même, la gender geography s'intéresse surtout aux femmes. Questionner la masculinité est presque iconoclaste (Longhurst, 2000). La géographie des homosexualités a pour objectif l'analyse des spatialités des homosexuels à différentes échelles et des stratégies spatiales qu'ils mettent en œuvre pour résister aux pouvoirs dominants qui assurent la régulation et la reproduction sociales. Mais elle permet aussi, en creux, de questionner les espaces de l'hétérosexualité, en particulier dans les villes.

\section{HOMOSEXUALITÉS ET TROPISME DES GRANDES VILLES}

Les géographes se focalisent sur le rapport particulier que les homosexuels entretiennent avec la grande ville car celle-ci est l'espace privilégié du déploiement de leurs cultures et de leurs modes de vie. Les métropoles sont attractives pour les gays et les lesbiennes car ils les considèrent comme des espaces-refuges.

\section{Des espaces pour les homosexuels}

La plupart des travaux ont fait de la ville occidentale leur terrain d'étude privilégié. Pourtant, des poèmes de Walt Whitman au Secret de Brokeback Mountain ${ }^{5}$, l'imaginaire gay se nourrit depuis longtemps des paysages de nature sauvage et des figures homoérotiques du fermier et du cow-boy (Haddock, 2008). Mais peu de géographes analysent les spatialités rurales des homosexuels. Ils insistent sur la

4. «Le postmodernisme en géographie ». 2004. Débat, L’Espace géographique, n 1/04, p. 6-37.

5. Adapté d'une nouvelle d'Annie Proulx, le film de Ang Lee, sorti en France en 2006, raconte une histoire d'amour impossible entre deux gardiens de bétail dans le Wyoming, des années 1960 à 1980. 
difficulté à vivre son homosexualité dans l'espace rural et sur l'isolement des homosexuels, entraînant souvent un véritable exode vers les villes (Bell, 2000 ; Bell et Valentine, 1995b ; Kramer, 1995 ; Weston, 1995). De même, les études sur le tourisme sont peu nombreuses. Gordon Waitt et Kevin Markwell (2006) en donnent deux explications : une institutionnalisation historique de l'hétérocentrisme et des lieux mis en tourisme par les homosexuels longtemps peu visibles ou seulement connus des initiés. Les différents auteurs montrent que le tourisme gay (le tourisme lesbien étant peu structuré) constitue un échappatoire à l' «interpellation hétérosexuelle » (Éribon, 1999, p. 88) et obéit à une logique de recherche de l'entre-soi. Il produit des territorialités spécifiques, presque exclusivement masculines (Hughes, 1997 ; Jaurand et Leroy, 2008 ; Puar, 2002 ; Waitt et Markwell, 2006). Si Emmanuel Jaurand (2005) s'attache à l'analyse des plages gays, les travaux sur le tourisme urbain dominent largement (Johnston, 2001 ; Rushbrook, 2002 ; Waitt et Markwell, 2006).

La ville focalise la plupart des regards pour plusieurs raisons. Premièrement, on peut y voir un effet d'école: les travaux nord-américains sur la géographie des homosexualités s'inscrivent pour partie dans le prolongement de ceux d'écologie urbaine, en particulier au travers de la question de la ségrégation socio-spatiale et de la régulation des différences dans l'espace public. Deuxièmement, ce thème de recherche, comme celui sur le genre, doit beaucoup aux mouvements politiques et à leurs pendants académiques, tant féministes qu'homosexuels, qui s'expriment essentiellement dans les grandes villes durant les années 1970 et 1980. En retour, le discours produit sur l'homosexualité par les chercheurs joue certainement un rôle performatif sur les mouvements homosexuels. Troisièmement, la géographie des homosexualités prend son essor avec l'apparition et le développement de l'épidémie de sida dans les grandes villes et avec la naissance des associations qui la combattent ${ }^{6}$. La maladie, en donnant à voir l'existence d'une «communauté » gay métropolitaine, légitime le thème de recherche.

La ville est l'espace dans lequel les homosexuels, notamment masculins, sont le plus visibles, même si concomitamment, c'est aussi un espace qui leur garantit l'anonymat, même relatif (Bell et Valentine, 1995a ; Binnie et Valentine, 1999 ; Ingram et $a l ., 1997)$. La géographie des homosexualités montre que la grande ville est une sorte de havre de paix et un espace de tolérance - au moins dans les représentations que s'en font les homosexuels. Mais elle l'est aussi souvent dans les pratiques, notamment pour ceux qui sont en rupture avec leur famille ou leur milieu professionnel. Dans Le danseur de Manhattan, roman sur le New York gay de la fin des années 1970, Andrew Holleran fait dire à l'un des personnages :

«La plupart d'entre nous avaient oublié qu'ils avaient une famille, à force de

6. Ainsi, l'association Act Up est fondée à New York en 1987 et à Paris en 1989. Ses modes d'action privilégient le lobbying et les performances, souvent spectaculaires, dans l'espace public, comme les diein lors de la Gay Pride par exemple. 
vivre entre folles à New York. Les familles appartenaient à ce passé indéchiffrable abandonné à l'ouest de l'Hudson (...) » ${ }^{7}$.

\section{Mirage urbain}

On peut donc avancer que le fait d'être homosexuel influence considérablement la décision de migrer et le choix du lieu de destination, en particulier pour les plus jeunes, au moment de la décohabitation familiale. Le fait que San Francisco soit devenue un haut lieu homosexuel doit tant aux mouvements de fuite et de repli des gays ${ }^{8}$ qu'elle a été comparée à un camp de réfugiés (Wittmann, 1992). Didier Éribon rappelle qu'il existe depuis toujours dans l'imaginaire gay « une véritable mythologie de la ville et de la capitale »(Éribon, 1999, p. 36). Les métropoles sont depuis longtemps attractives pour les homosexuels, surtout masculins (Bech, 1997), fait historique déjà bien étudié (Aldrich, 2006 ; Tamagne, 2000). John D’Emilio (1983) insiste sur le rôle de l'urbanisation et de l'industrialisation des pays occidentaux dans les migrations des homosexuels de l'espace rural vers la ville à la fin du $19^{\mathrm{e}}$ siècle et au début du $20^{\mathrm{e}}$. On peut penser que ces migrations, à l'instar des autres, se sont accentuées avec l'augmentation de la mobilité ces dernières décennies. A contre-courant des travaux qui montrent le tropisme des grandes villes en questionnant l'identité, l'expérience et le vécu des gays et des lesbiennes, Marianne Blidon (2007) minimise l'importance actuelle de ces migrations en France, à partir de l'analyse de la relation entre taille des villes et nombre de commerces homosexuels. Pourtant, ses résultats tendraient à montrer leur surreprésentation systématique dans les plus grandes villes. L'auteur indique toutefois les limites de son étude: le degré de fréquentation des commerces est inconnu, les données ne sont pas exhaustives et la définition d'un établissement gay demeure floue. Les migrations sont aussi bien pendulaires (Pollak, 1982) que de longue durée ou définitives (Knopp et Brown, 2003 ; Weston, 1995). Si la métaphore du voyage et la recherche d'un paradis (sexuel) perdu sont au cour de la culture gay (Jaurand et Leroy, 2008), c'est bien «la force d'attraction - réelle et imaginaire - exercée par la ville qui reste le phénomène qui concerne le plus grand nombre » (Éribon, 1999, p. 37).

La cartographie de la part des couples de même sexe dans l'ensemble des couples aux États-Unis met en évidence de fortes concentrations résidentielles d'homosexuels dans certains quartiers des métropoles et dans quelques stations balnéaires (Gates et Ost, 2004). Ainsi, dans le quartier de Castro, à San Francisco, un

7. Holleran, A. 2003. Le danseur de Manhattan, Paris, 10/18, p. 271.

8. De nombreux marins, chassés de l'armée américaine durant la Seconde Guerre mondiale à cause de leur homosexualité, ne rentrèrent pas chez eux par crainte des réactions homophobes et pour échapper à un mariage non voulu. Ils restèrent à San Francisco, lieu de leur démobilisation et y construisirent un espace de liberté, le quartier de Castro (Bérubé, 1991). 
calcul montre qu'en 2000, près de la moitié des couples étaient des couples de même sexe, très majoritairement des hommes. Comme par ailleurs, différentes enquêtes indiquent que le nombre de gays vivant en couple est faible (Sears et al., 2005), cette forte concentration dans des secteurs peu étendus des métropoles témoigne de la recherche de l'entre-soi des gays aux États-Unis. Si le besoin d'entre-soi et la recherche de centralité et d'urbanité sont plus faibles chez les lesbiennes, est-ce parce que l'espace périurbain voire rural est un cadre qui convient mieux à celles qui élèvent des enfants, parce que leurs revenus sont moins importants ou parce qu'elles ont moins besoin de multiplier les rencontres et les partenaires sexuels? Les études sont trop peu nombreuses pour privilégier l'une de ces hypothèses. A la suite de Castells (1983), d'autres chercheurs analysent le rôle moteur des homosexuels dans les transformations sociales, économiques et spatiales de plusieurs grandes villes nord-américaines et dans les processus de renaissance urbaine, notamment la gentrification (Knopp, 1992 ; Lauria et Knopp, 1985 ; Rothenberg, 1995). Ils constituent souvent des groupes pionniers à la recherche de «niches » spatiales dans la ville.

Que l'on s'entende : il ne faut pas imaginer que la mobilité résidentielle de cette population s'effectue uniquement de l'espace rural ou des petites villes vers les métropoles. Non seulement les «campagnes » sont aujourd'hui bien trop dépeuplées pour constituer un stock important de migrants potentiels ${ }^{9}$, mais la mobilité n'est pas systématiquement linéaire (Gorman-Murray, 2007). Si on peut affirmer que l'opposition symbolique entre l'urbain et le rural est essentielle dans le processus de coming out (ou « sortie du placard »), il y a des tours et des détours multiples voire des retours dans nombre de trajectoires individuelles. Comme les autres populations, les gays et les lesbiennes alimentent des flux de migrations multiscalaires et polymorphes: internationales, interrégionales, interurbaines et intraurbaines (Knopp et Brown, 2003). Il faut insister sur le rôle déterminant de l'homophobie et de l'injure dans la fuite éventuelle vers les métropoles. L'injure est une expérience partagée par les homosexuels dans tous les espaces (public, domestique, scolaire, professionnel, sportif, etc.) et qui pèse sur la conscience (et la haine ?) qu'ils ont d'eux même (Éribon, 1999). Mais on peut penser que d'autres facteurs, en particulier économiques, et à certaines périodes de la vie, participent de la décision de migrer vers la ville. Il n'empêche que la notion de déplacement est centrale dans la culture gay. Est-ce à dire, comme l'avance Henning Bech (1997), que pour les homosexuels, surtout masculins, il est plus important de chercher un lieu que de le trouver?

9. Au contraire, les espaces « ruraux» proches des grandes villes ont aujourd'hui un solde migratoire positif. 


\section{LE « DROIT À LA VILLE » ${ }^{10}$ POUR LES HOMOSEXUELS}

La plupart des travaux géographiques traitent de la dichotomie visibilité/invisibilité. Certains se focalisent sur les espaces que les gays et les lesbiennes s'approprient ou les performances (exhibitions, démonstrations, rôles) qui les rendent visibles, d'autres sur l'invisibilité et la transgression, avec le détournement de certains lieux publics, ou le caractère contraint des pratiques de la ville des homosexuels.

\section{La visibilité gagnée dans la ville}

De nombreux auteurs interrogent la genèse, la forme et l'usage des espaces appropriés par les homosexuels dans les métropoles occidentales (Binnie, 1995 ; Brown, 2000 ; Ingram et $a l .$, 1997). Bien que ces quartiers soient des espaces de consommation, structurés par des commerces destinés aux homosexuels, et des espaces touristiques participant de l'internationalisation de la culture gay, ils ne peuvent être réduits à ces deux fonctions. En effet, les différents travaux soulignent le rôle majeur de ces territoires - produits de la stigmatisation - dans la construction spatiale des identités homosexuelles ou dans l'élaboration d'un «référent géographique » de ces identités, pour reprendre la formule de Mathis Stock (2006, p. 19). Ils rendent visibles les homosexuels et permettent à certains d'entre eux, en particulier les plus jeunes, de faire leur coming out (Valentine et Skelton, 2003). De plus, ils augmentent les possibilités de rencontre, à l'intérieur et à l'extérieur des établissements commerciaux ${ }^{11}$, et les homosexuels y valident leur identité au contact du même. Bien sûr, personne n'y est confiné : ces quartiers fonctionnent comme des points de passage, comme cela a été montré avec le Marais, le quartier gay de Paris (Leroy, 2005). Et même pour les homosexuels qui ne les fréquentent pas ou plus, leur existence est rassurante. Ces quartiers sont des constructions essentiellement masculines ${ }^{12}$. Dans leur majorité, les lesbiennes s'identifient peu à des espaces spécifiques. Cet aspect mériterait un long développement, impossible à mener dans les limites de cet article. On rappelle juste que plusieurs études montrent que les spatialités lesbiennes sont plus réticulaires qu'aréales, ou plus dispersées, et aussi davantage éphémères (Podmore, 2006 ; Valentine, 1995). Dans le cas extrême de Los Angeles, le droit à la ville pour les homosexuels est devenu le droit d'une ville, avec la création en 1984 par les communautés gays et lesbiennes d'une municipalité autonome, West Hollywood (Forest, 1995).

10. Expression empruntée à Henri Lefebvre (1968).

11. Les stratégies développées par les homosexuels pour se reconnaître dans l'espace public, et en particulier le rôle du regard (gaze), ne sont pas développées ici. Sur cette question, voir Bech, 1997.

12. En 2005, 97\% des commerces gays et lesbiens du Marais étaient destinés aux seuls hommes (Leroy, 2005). 
Une autre série de travaux questionne la visibilité homosexuelle au travers du corps - considéré comme le «site » de formation de l'identité et le vecteur principal de la relation entre l'individu et l'espace (Brown, 2000 ; Duncan, 1996). Les performances hétérosexuelles qui se répètent dans l'espace public, au travers des pratiques et des discours, font croire qu'il est depuis toujours naturellement hétérosexuel - alors qu'il est le produit de la hiérarchie entre les sexualités (Browne, 2007). C'est pourquoi, de nombreux chercheurs montrent l'importance des performances du corps homosexuel dans l'espace public pour résister à l'hétérosexisme et à l'homophobie (Bell et al., 2001). Ainsi, la Gay Pride, les Gay Games et les autres manifestations dans les rues des grandes villes constituent des appropriations éphémères de l'espace public et des formes de résistance à l'hétéronormativité qui l'organise (Brickell, 2000 ; Johnston, 2001 ; Waitt, 2003). Ces rassemblements cherchent à l'homosexualiser en rendant publics et visibles les corps gays et lesbiens, souvent condamnés à demeurer privés et invisibles. A l'occasion de ces manifestations, on reproche parfois aux homosexuels d'exposer leur préférence sexuelle, parfois de manière provocante. Lynda Johnston (2001) suggère au contraire que ces manifestations de visibilité homosexuelle sont d'un grand intérêt pour les hétérosexuels. En effet, quand l'homosexuel est physiquement défini, que son corps apparaît différent par divers artifices, la frontière devient clairement visible entre lui et l'hétérosexuel et donc moins menaçante pour le groupe dominant. A l'instar des autres déviants, quand l'homosexuel est démasqué, il devient plus aisé de le stigmatiser (Goffman, 1975).

\section{Territoires de l'interdit, interdits de territoire}

La grande ville n'est pas un milieu homogène. Cela signifie que la visibilité n'est jamais totale ni acquise pour toujours - comme l'anonymat. Des recherches se focalisent sur des manières différentes de contester et de transgresser la norme dans l'espace urbain. Elles interrogent l'organisation et le rôle des lieux de drague et d'interactions sexuelles anonymes entre hommes (Binnie, 2001 ; Gaissad, 2006 ; Humphreys, 2007). Elles montrent que ces espaces publics (parcs, quais, cimetières, parkings, toilettes publiques, chantiers, etc.), souvent éloignés des centres pour des raisons de discrétion et essentiellement fréquentés le soir et la nuit, sont détournés de leur usage principal de manière illicite. La pratique très codifiée de ces lieux, souvent ignorée des non-initiés, dans lesquels les regards et les gestes remplacent la parole, questionne les notions d'altérité, d'appropriation spatiale et d'intimité. Avec ce type de lieu fondé par l'interdit, les pratiques des homosexuels masculins sont rationalisées, leurs espaces fonctionnalisés et leur temps rentabilisé (Pollak, 1982). En rupture avec les modes de vie hétérosexuels, souvent considérés par les gays comme contraignants 
parce que caractérisés par des relations de couple durables, en opposition avec la mise en scène de soi dans les espaces de visibilité, ces pratiques participent également de la construction des identités homosexuelles (Leroy, 2005). Si certains de ces lieux sont mythiques dans l'imaginaire et la culture gays, David Bell (2001) avance que toutes les rues constituent potentiellement des zones érotiques voire érogènes. N'est-ce pas l'ensemble de la grande ville qui est un marché sexuel à ciel ouvert pour les gays ?

Il y a donc des lieux de l'interdit, dans lesquels les homosexuels ne cherchent pas la visibilité. Il y en a d'autres où elle est impossible. Dans l'espace public construction sociale dotée d'une forte dimension normative (Bondi et Rose, 2003) - les homosexuels doivent régler leur comportement en utilisant différentes stratégies. Michel de Certeau (1990) a bien montré leur importance lorsqu'il faut pratiquer les territoires des «Autres». Elles varient selon les trajectoires de vie de chacun et surtout en fonction des lieux pratiqués. Par exemple, se tenir par la main dans la rue demeure aujourd'hui pour un couple de même sexe un acte politique audacieux voire dangereux. Une enquête portant sur l'identification des espaces publics parisiens dans lesquels des contacts physiques entre les membres d'un couple de même sexe sont envisageables ou effectués a révélé un fort gradient $d u$ «centre gay », le Marais, vers la périphérie (Cattan et Leroy, 2008). Il y a les espaces du possible, le quartier gay uniquement, ceux du peut-être (sa proche périphérie) et ceux de l'impossible (tout le reste de l'espace public). On retrouve la typologie des lieux pratiqués par le stigmatisé proposée par Erving Goffman (1975) : les lieux retirés, policés et interdits.

Il identifie différentes stratégies nécessaires aux individus stigmatisés pour ajuster leur attitude et pratiquer l'espace public et plus généralement la ville : dissimulation, invention de «désidentificateurs » (se faire passer pour ce que l'on n'est pas), maintien à distance, résistance, dévoilement (Goffman, 1975). En fonction du contexte social et spatial, elles sont tour à tour utilisées par les homosexuels pour contrôler l'information relative à leur identité sexuelle. L'évaluation de la bonne distance à établir entre eux et les « Autres » est primordiale. Elle varie selon le degré de connaissance et d'intimité, et selon la situation et le lieu. La seule différence entre la grande ville et les autres espaces est que cette distance est beaucoup plus courte dans la grande ville, d'où son attractivité pour les homosexuels. De plus, si le dévoilement est possible dans les espaces publics et privés appropriés par les homosexuels et lors des manifestations de rue, la visibilité reste déterminée par les normes imposées par la majorité hétérosexuelle et généralement intériorisées par les gays et les lesbiennes. Leur transgression a un coût. Ainsi, en 2007, dans le XVIII ${ }^{\mathrm{e}}$ arrondissement de Paris, une quinzaine d'adolescents extrait d'un bus de nuit deux jeunes hommes qui viennent de se faire «un petit bisou». Ils les frappent très violemment en hurlant «à mort, sales pédés ! ». L'un des agressés explique :

«On aurait dit que c'était leur récréation... Je constate que quand t'es pédé, t'es 
pas libre. Il y a des quartiers où tu ne peux pas vivre ouvertement ta vie amoureuse. A tout moment tu peux te faire démonter ${ }^{13}$ (souligné par moi).

L'utilisation souvent obligée de la stratégie de dissimulation par les homosexuels contribue à renforcer l'hétéronormativité de l'espace public (Kirby et Hay, 1997). Elle témoigne de «l'immense puissance des dispositifs spatiaux dans les manières de discipliner les corps et de fabriquer le corps social lui-même » (Chivallon, 2008, p. 85).

\section{CONCLUSION}

La grande ville occidentale est un espace de liberté, même relative, pour les gays et les lesbiennes, surtout lorsque s'y est développé un quartier qui les rend visibles et maximise les possibilités de rencontre. Au travers des espaces qu'ils détournent ou s'approprient, des réseaux de sociabilité et de solidarité qu'ils tissent par l'intermédiaire des commerces et des associations, des manifestations publiques dans lesquelles ils utilisent leur corps comme outil de résistance, et parce qu'ils partagent l'expérience de la stigmatisation, les homosexuels peuvent développer dans la ville une culture commune et une identité collective. Bien sûr, ces espaces appropriés peuvent être intimidants, en particulier pour ceux qui ne sont pas sortis du placard, générer de l'exclusion, ou agir comme des repoussoirs sur ceux qui ne veulent ni s'identifier ni être identifiés à un territoire ou à un style de vie. Avec cette (r)évolution de la place des homosexuels dans la ville, impulsée par les mouvements politiques et associatifs, ne passe-t-on pas de l'impératif de l'invisibilité à l'injonction à la visibilité (Chauncey, 2003) ? D'ailleurs, certaines petites annonces de rencontres entre gays sont remplies de descriptions telles que «hors ghetto», «hors Marais» ou «look hétéro ». Elles témoignent d'une diversité des modes de vie mais aussi, peut-être, d'une homophobie redirigée vers soi (Leroy, 2005).

L'orientation sexuelle participe aux processus de stigmatisation et de relégation des individus à toutes les échelles, peut-être autant que les critères traditionnels de classes et de races. Même dans les grandes villes, l'espace public demeure inégalement accessible. Il est organisé par et pour la domination de l'hétérosexualité. Cela démontre l'efficacité de l'espace dans l'organisation et la perpétuation de l'ordre social et des hiérarchies créées et naturalisées par les sociétés, et donc l'intérêt pour la géographie de traiter des questions de pouvoir et de domination, mais aussi d'altérité. Aujourd'hui, le droit à la visibilité des homosexuels dans la ville est encore strictement limité à ce que la norme autorise. Il est circonscrit aux seuls quartiers gays (et souvent seulement à la tombée du jour), ce qui démontre toute leur importance dans les pratiques et les 
représentations spatiales des homosexuels, justifie la recherche d'un entre-soi relatif et donne un avantage à la métropole comme lieu de vie et d'épanouissement. Mais cette assignation spatiale de fait n'est-elle pas symptomatique d'une société qui sécrète des espaces de liberté mais les confine pour mieux les contrôler? Entre visibilité et invisibilité, exposition et anonymat, c'est toujours une double vi(ll)e que connaissent les gays et les lesbiennes, en fonction des différentes sphères dans lesquels ils évoluent. C'est seulement à la condition de posséder une connaissance aiguisée des limites et des frontières à ne pas franchir qu'ils ont la possibilité d'une ville.

\section{RÉFÉRENCES BIBLIOGRAPHIQUES}

ALDRICH, R. 2006. Une histoire de l'homosexualité, Paris, Seuil.

BECH, H. 1997. When Men Meet: Homosexuality and Modernity, Cambridge, Polity.

BELL, D. 2000. «Farm boys and wild men: rurality, masculinity and homosexuality », Rural Sociology, vol. 65, $\mathrm{n}^{\circ} 44$, p. 547-561.

BELL, D. 2001. «Fragments for a Queer City», dans D. Bell et al., Pleasure Zones. Bodies, Cities, Spaces, Syracuse, Syracuse University Press, p. 84-102.

Bell, D. ; Binnie, J. ; Holliday, R. ; Longhurst, R. ; Peace, R. 2001. Pleasure Zones. Bodies, Cities, Spaces, Syracuse, Syracuse University Press.

Bell, D. ; Valentine, G. (dir.) 1995a. Mapping Desire: geographies of sexualities, Londres, Routledge.

Bell, D. ; Valentine, G. 1995b. «Queer country: Rural Lesbian and Gay Lives », Journal of Rural Studies, vol. 11, n², p. 113-122.

BÉrubé, A. 1991. Coming Out Under Fire. The History of Gay Men and Women in World War Two, New York, Plume.

BINNIE, J. 1995. «Trading places. Consumption, sexuality and the production of queer space », dans D. Bell ; G. Valentine (dir.), Mapping Desire: geographies of sexualities, Londres, Routledge, p. 182-199.

BINNIE, J. 2001. «The Erotic Possibilities of the Cities », dans D. Bell et al., op. cit., p. 103-130.

Binnie, J. ; VAlEntine, G. 1999. «Geographies of sexuality - a review of progress », Progress in Human Geography, vol. 23, n², p. 175-187.

BLIDON, M. 2007. «Ville et homosexualité, une relation à l'épreuve de la cartographie », dans M.-F. Mattei ; D. Pumain (coord.), Données Urbaines 5, Paris, Anthropos, p. 67-76.

BLIDON, M. 2008. «Jalons pour une géographie des homosexualités », L'Espace géographique, tome $37, \mathrm{n}^{\circ} 2$, p. 175-189.

BondI, L. ; Rose, D. 2003. «Constructing gender, constructing the urban: a review of 
Anglo-American feminist urban geography », Gender, Place and Culture, vol. 10, $\mathrm{n}^{\circ}$, p. 229-245.

BRICKELL, C. 2000. «Heroes and invaders: gay and lesbian pride parades and the public/private distinction in New Zealand media accounts », Gender, Place and Culture, vol. $7, \mathrm{n}^{\circ} 2$, p. 163-178.

Brown, M. 2000. Closet space: geographies of metaphor from the body to the globe, Londres, Routledge.

BROWNE, K. 2007. "(Re)making the other, heterosexualising everyday space », Environment and Planning A, vol. 39, n²4, p. 996-1014.

ButLer, J. 2005. Trouble dans le genre, Paris, La Découverte [1990].

CASTELLS, M. 1983. "Cultural identity, sexual liberation and urban structure: the gay community in San Francisco », dans M. Castells (dir.), The City and the Grassroots: A Cross-Cultural Theory of Urban Social Movements, Londres, Edward Arnold, p. 138170.

CAtTAN, N. ; LeRoy, S. 2008. "Gender in public space: beyond the dominant meaning of the urban », communication, 2008 Association of American Geographers Meeting, Boston, 15-19 avril 2008.

Certeau, M. de 1990. L'invention du quotidien. 1. Arts de faire, Paris, GallimardFolio.

ChaunceY, G. 2003. Gay New York, 1890-1940, Paris, Fayard [1995].

Chivallon, C. 2008. «L'espace, le réel et l'imaginaire : a-t-on encore besoin de la géographie culturelle ? », Annales de géographie, n660-661, p. 67-89.

Cusset, F. 2003. French Theory, Paris, La Découverte.

Di MÉo, G. 2004. «Composantes spatiales, formes et processus géographiques des identités », Annales de Géographie, n639, p. 339-362.

D’Emilio, J. 1983. Sexual Politics, Sexual Communities: The Making of a Homosexual Minority in the United States, 1940-1970, Chicago, University of Chicago Press.

DunCAN, N. (dir.) 1996. Bodyspace: Destabilizing Geographies of Gender and Sexuality, Londres, Routledge.

ÉRIBON, D. 1999. Réflexions sur la question gay, Paris, Fayard.

FOREST, B. 1995. "West Hollywood as symbol: the significance of place in the construction of gay identity », EPD : Society and Space, vol. 13, n², p. 133-157.

FrIOUX, D. 2003. «Universalisme/Différentialisme», dans L.-G. Tin (dir.), Dictionnaire de l'Homophobie, Paris, PUF, p. 413-415.

GAISSAD, L. 2006. Une forme notoire de sexualité secrète : chronique territoriale du désir entre hommes dans le sud de la France, thèse de doctorat de sociologie, Université de Toulouse II-Le Mirail.

Gates, G.J. ; Ost, J. 2004. The Gay and Lesbian Atlas, Washington D.C., The Urban 
Institute Press.

GofFMAN, E. 1975. Stigmate, Paris, Minuit [1963].

Gorman-Murray, A. 2007. «Rethinking queer migration through the body », Social \& Cultural Geography, vol. 8, n¹, p. 105-121.

HADDOCK, B.H. 2008. « Rough and Ready: The Gay Male Image in Rural America », communication, 2008 Association of American Geographers Meeting, Boston, 15-19 avril 2008.

HuBBARD, P. 2000. «Desire/disgust: mapping the moral contours of heterosexuality », Progress in Human Geography, vol. 24, n², p. 191-217.

Hughes, H. 1997. «Holidays and Homosexual Identity», Tourism Management, vol. $18, \mathrm{n}^{\circ} 1$, p. 3-7.

HuMPHREYS, L. 2007. Le commerce des pissotières: Pratiques homosexuelles anonymes dans l'Amérique des années 1960, Paris, La Découverte [1970].

IngRam, G.B.; Bouthillette, A.-M.; RetTer Y. (dir.) 1997. Queers in Space: Communities, Public Places, Sites of Resistance, Seattle, Bay Press.

JAURAND, E. 2005. «Territoires de mauvais genre? Les plages gays », Géographie et Cultures, ${ }^{\circ} 54$, p. 71-84.

JAURAND, E. ; LEROY, S. 2008. «Le littoral : un paradis gay? », actes du colloque Le Littoral : Subir, Dire, Agir, Lille, IFRESI-MESHS-CNRS, cd-rom, 15-18 janvier 2008.

Johnston, L. 2001. «(Other) Bodies and Tourism Studies», Annals of Tourism Research, vol. $28, \mathrm{n}^{\circ} 1$, p. $180-201$.

KIRBY, S. ; HAY, I. 1997. "(Hetero)sexing Space: Gay Men and "Straight" Space in Adelaide, South Australia », The Professional Geographer, vol. 49, n³, p. 295-305.

KNOPP, L. 1992. «Sexuality and the spatial dynamics of capitalism », EPD : Society and Space, vol. 10, n6, p. 651-669.

KnopP, L. ; Brown, M. 2003. «Queer diffusions », EPD : Society and Space, vol. 21, $\mathrm{n}^{\circ} 4$, p. 409-424.

KRAMER, J.L. 1995. «Bachelor farmers and spinsters: lesbian and gay identity and community in rural North Dakota», dans D. Bell ; G. Valentine (dir.), op. cit., p. 200213.

LAURIA, M. ; KNOPP, L. 1985. "Towards an analysis of gay communities in the urban renaissance », Urban Geography, vol. 6, n², p. 152-169.

LEROY, S. 2005. «Le Paris gay. Eléments pour une géographie de l'homosexualité », Annales de géographie, n646, p. 579-601.

LONGHURST, R. 2000. "Geography and gender: masculinities, male identity and men », Progress in Human Geography, vol. 24, n³, p. 439-444.

PODMORE, J.A. 2006. "Gone 'underground'? Lesbian visibility and the consolidation of queer space in Montréal », Social \& Cultural Geography, vol. 7, n4, p. 595-625. 
PollaK, M. 1982. «L'homosexualité masculine, ou : le bonheur dans le ghetto ?», Communications, $\mathrm{n}^{\circ} 35$, p. 37-55.

PUAR, J.K. 2002. «Circuits of Queer Mobility. Tourism, Travel and Globalization », $G L Q$, vol. 8, n¹-2, p. 101-137.

RocCHI, J.-P. 2003. «Hétérosexisme », dans D. Éribon (dir.), Dictionnaire des cultures Gays et Lesbiennes, Paris, Larousse, p. 243-244.

RothenberG, T. 1995. " "And she told two friends": Lesbians creating urban social space », dans D. Bell ; G. Valentine (dir.), op. cit., p. 165-181.

RushbrooK, D. 2002. «Cities, Queer Space, and the Cosmopolitan Tourist», GLQ, vol. 8, $\mathrm{n}^{\circ} 1-2$, p. 183-206.

Sears, R.B.; Gates, G. ; Rubenstein, W.B. 2005. Same-sex Couples and Same-sex Couples raising children in the United States, Los Angeles, The Williams Project/UCLA School of Law.

STOCK, M. 2006. "L'hypothèse de l'habiter poly-topique: pratiquer les lieux géographiques dans les sociétés à individus mobiles », EspacesTemps.net, Textuel, http://espacestemps.net/document1853.html

Tamagne, F. 2000. Histoire de l'homosexualité : Berlin, Londres, Paris, 1919-1939, Paris, Seuil.

TAmagne, F. 2006. «Histoire des homosexualités en Europe : un état des lieux », Revue d'Histoire Moderne et Contemporaine, vol. 53, n 4, p. 7-31.

VALENTINE, G. 1995. «Out and about: a geography of lesbian communities », International Journal of Urban and Regional Research, vol. 19, $\mathrm{n}^{\circ} 1$, p. 96-111.

Valentine, G. ; Skelton, T. 2003. «Finding Oneself, Losing Oneself: The Lesbian and Gay 'Scene' as a Paradoxical Space », International Journal of Urban and Regional Research, vol. 27, n4, p. 849-866.

WAITT, G. 2003. «Gay Games: performing 'community' out from the closet of the locker room », Social \& Cultural Geography, vol. 4, n², p. 167-183.

WaitT, G. ; Markwell, K. 2006. Gay Tourism: Culture and Context, New York, Haworth Hospitality Press.

Weston, K. 1995. "Get Thee to a Big City: Sexual Imagery and the Great Gay Migration », GLQ, vol. 2, n³, p. 253-277.

Wittmann, C. 1992. «A Gay Manifesto », dans K. Jay ; A. Young (dir.), Out of the Closets. Voices of Liberation, New York, New York University Press, p. 330-342. 